\title{
LabVIEW Implementation of WSN for Real Time Monitoring in Precision Agriculture
}

\author{
Shivangi Gupta \\ Assistant Professor \\ MBSCET, Jammu (India)
}

\author{
Lini Mathew, PhD \\ Associate Professor \& Head \\ NITTTR, Chandigarh ( India)
}

\begin{abstract}
Agriculture is the backbone of any country's economy and there is a strong association between agricultural growth and economic prosperity. In spite of that, exposure of agriculture to advancement of technologies is inadequate in India. New and effective technology is required to improve continuously productivity, sustainability profitability for our major farming systems. Development and expansion in wireless sensor network made this possible which was nearly impossible few decades ago .In this paper, real -time data is collected using wireless sensor nodes and transmitted to base station using ZigBee wireless technology. The data is received and saved to achieve soil moisture, temperature and humidity, and soil ph value of individual nodes placed at field station. The values are graphically displayed and analyzed at base station using LabVIEW which can be useful for taking various types of decisions for farming practice. This system has the advantage of flexible networking and it overcomes the limitation of wired sensor networks.
\end{abstract}

\section{General Terms}

WSN, Precision Agriculture, Nodes

\section{Keywords}

Precision Agriculture, ZigBee, LabVIEW , Transmitting Nodes, Receiving Node, Field station, Base station

\section{INTRODUCTION}

Precision Agriculture refers to use of information and control technologies in agriculture [1]. It has new and rising promising communications technologies which is key requirement in this application to improve the irrigation system of Indian agriculture. It leads to higher yields and lower costs and also provides adequate irrigation in particular area. Another name for precision agriculture is crop management or Site-Specific Management. Precision agriculture makes sure that all kind of variations occurring in crop or soil properties are noted and mapped properly in order to have better quality control of the produce and necessary actions are also taken. On the theoretical point of view, precision agriculture can be considered to have three-phases.

The first phase is data collection. It also comprises of measuring (which can be more or less automated) physical parameters describing the soil and the growing crops. The second phase is the data interpretation. This step is not yet automated, that means the decision making devolves to the farmer, who has to make choices. The third phase is the application. It involves the modulation of fertilizers doses, adjustment of sowing parameters, the site-specific application of herbicides, etc.

\subsection{The Need for Precision Agriculture}

Today, most crucial problems faced by farmers in India is lack of scientific knowledge of agriculture parameters like level of moisture in soil, presence of percentage of minerals available in soil and less knowledge of the type of fertilizers to be added which actually hits the growth and yield of crop. With the evolution of WSN, it becomes possible to use them for automatic environment monitoring and controlling the field parameter for precision agriculture application. So, therefore Precision irrigation can act as an important practice in water-saving agriculture cropping system, which allows producers to maximize their productivity while saving water [2]. Till today despite the efforts of the Govt.. of India, analysis of soil could not be implemented on the ground on the large scale, for the reason of lack of appropriate number of labs available in the rural area and further cost involved in the complete analysis of soil is also on the higher side. Further sending one or two samples could not tell the actual position of the contents of the whole field. A precision farming approach recognizes site-specific differences within fields and adjusts management actions accordingly instead of managing an entire field based upon some hypothetical average condition, which may not exist anywhere in the field, So precision agriculture refers to a set of technologies that introduced the concept of the local variation into the large scale mechanization, which is essential to large fields [3].

\subsection{Precision Agriculture and WSN}

In the past few years, new trends have emerged in the agricultural sector. Precision agriculture (PA) is applied using WSN. Wireless sensor network WSN consists of spatially distributed sensor [4] and nodes which concentrate on providing the means for observing, assessing and controlling agricultural practices. WSN system for PA requires a centralized control unit with user interface, communication gateways and router, power element and the most importantly are the sensor. [5] It concerns as well pre- and post-production aspects of agricultural enterprises.

\subsection{WSN Tool : ZigBee}

ZigBee is a emerging wireless technology with low power needs and low data rate capabilities which, perfectly suits precision agriculture. It is also known as LP-WPAN (Low Power-Wireless Personal Area Network) It is characterized by a short range of few hundred meters and defined rate of $250000 \mathrm{bit} / \mathrm{s}$. The standard was conceived to inter-connect embarked units like sensors. It is based on the standard IEEE 802.15.4 . ZigBee can work on three frequency bands: $2.4 \mathrm{GHz}$ (INDIA), $868 \mathrm{MHz}$ (Europe) and $915 \mathrm{MHz}$ (North America) .

ZigBee standard supports three types of device support to it system: ZigBee Coordinator, ZigBee Router and ZigBee End Device.[6][7][8].

- ZigBee Coordinator (ZC) is at the top in topology and there should be one in each network. It initiates the ZigBee network, select frequency to be used by the network and allow devices to connect.

- ZigBee Router (ZR) acts as a intermediate between Nodes or ZigBee End devices(ZED) and ZC.

- $\quad$ ZigBee End devices (ZED) report sensor states. 


\section{SYSTEM DESIGN}

Our system consists of four ZigBee modules placed as four different nodes creating WSN for precision agriculture (see Figure 1). ZigBee placed at nodes 1, 2, 3 are ZigBee end
devices(ZED) and ZigBee placed at reciver node is the ZigBee corrdinator (ZEC). Follwing is the description of component invole in our system.
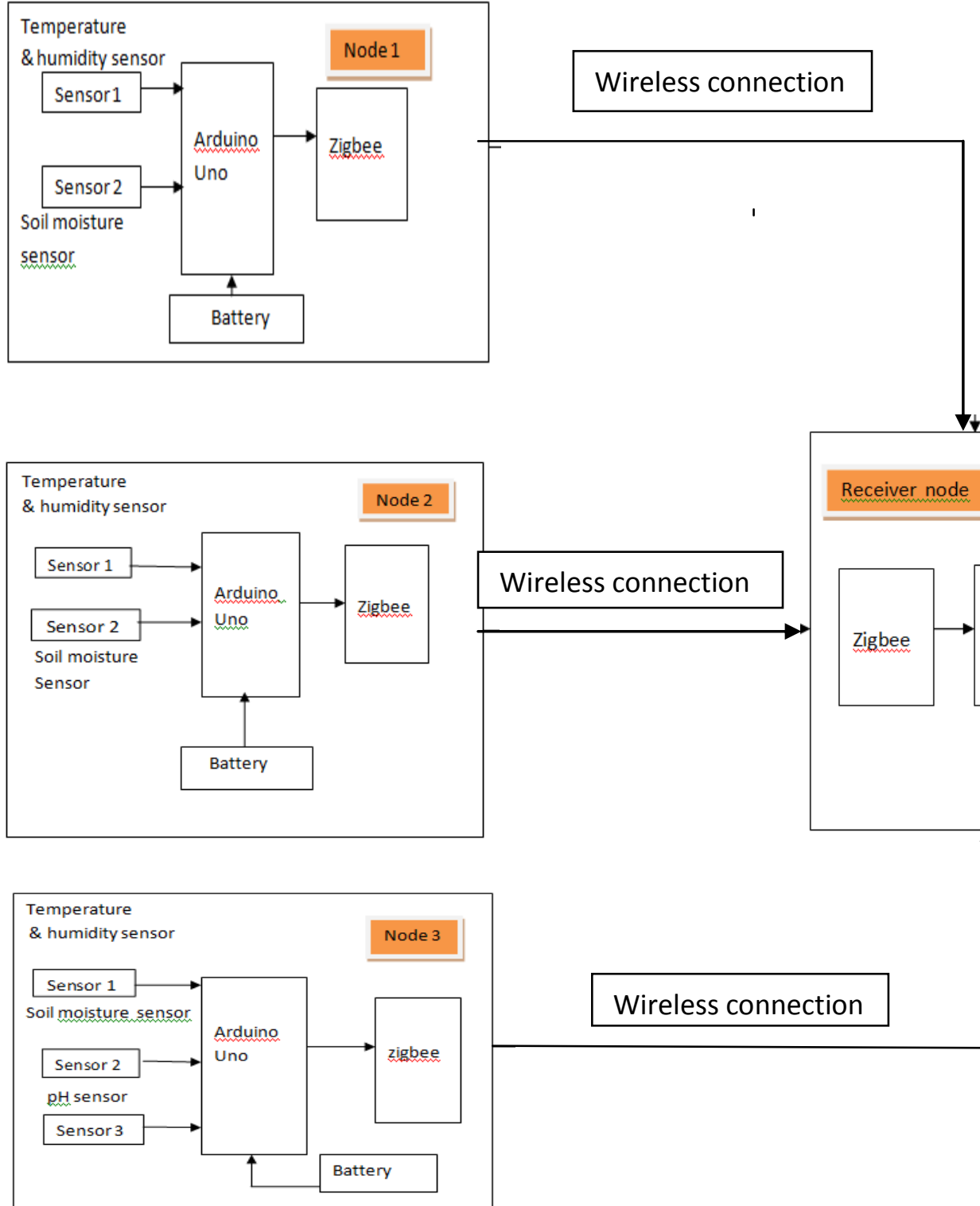

Wireless connection

Fig 1: Block diagram of ZigBee based precision agriculture

\subsection{Arduino Uno and Atmega328 Microcontroller}

The Arduino Uno board is based on the ATmega328. It has 14 digital input/output pins, 6 analog inputs, a $16 \mathrm{MHz}$ crystal oscillator, a USB connection, a power jack, an ICSP header, and a reset button. Arduino receive input data from a variety of sensors and can affect its surroundings by controlling smart agriculture, homes, smart classrooms and any other smart system. Power required for Arduino Uno is 5 volts and clock speed of microcontroller is $16 \mathrm{MHz}$ The microcontroller on the board is programmed using the Arduino programming language ATmega328 is basic component on Arduino Uno The Atmel picoPower ATmega328/P is a low-power CMOS 8-bit microcontroller based on the AVR® enhanced RISC architecture. ATmega328/P achieves throughputs close to $1 \mathrm{MIPS}$ per $\mathrm{MHz}$ by executing powerful instructions in a single clock cycle.

\subsection{Humidity and Temperature Sensor}

This DHT11 Humidity and Temperature Sensor feature a temperature \& humidity sensor complex with a calibrated digital signal output. For technical specification of DHT11 Temperature and humidity sensor (see Table 1). By using 
temperature \& humidity sensing technology and the exclusive digital-signal-acquisition technique, it ensures excellent longterm stability and high reliability.

Table 1. DHT11 Technical Specifications

\begin{tabular}{|l|l|l|l|}
\hline Item & Parameter & Range & Accuracy \\
\hline DHT11 & Humidity & $20-90 \%$ & $\pm 5 \%$ RH \\
\hline DHT11 & Temperature & $0-50^{\circ} \mathrm{C}$ & $\pm 2^{0} \mathrm{C}$ \\
\hline
\end{tabular}

When this sensor is connected to a high-performance 8-bit microcontroller as such sensor having a resistive-type humidity measurement component and an NTC temperature measurement component, then it offers excellent quality, fast response, anti-interference ability and cost-effectiveness.

When the power being supplied to the sensor, we should not send any instruction to the sensor within one second in order to pass the unstable status. One capacitor of $100 \mathrm{nF}$ can be added between VDD and GND for power filtering. Each DHT11 element has has four pins. Connect the first pin on left of sensor ('VCC') to $5 \mathrm{~V}$ of arduino board, the second pin ('Data') to digital pin 2 of arduino, third pin is not used and the fourth pin ('GND') to ground of arduino.

\subsection{Soil Moisture Sensor}

This soil moisture sensor measures the volumetric content of water inside the soil and gives us the moisture level as output. The sensor is FC-28 module. The sensor is equipped with both analog and digital output, so it can be used in both analog and digital mode but analog output is more accurate. Power requirement for this sensor is $3.3-5 \mathrm{~V}$. We are using it in analog mode.

Two probes of soil moisture sensor are actually used for measuring volumetric water content. The two probes allow the current to pass through the soil and then it gets the resistance value to measure the moisture value. When there is more water, the soil will conduct more electricity which means that there will be less resistance. Therefore, the moisture level will be higher. Dry soil conducts electricity poorly, so when there will be less water, then the soil will conduct less electricity which means that there will be more resistance. Therefore, the moisture level will be lower. The Module also contains a potentiometer which will set the threshold value and then this threshold value will be compared by the LM 293 comparator attached to it. The output LED will light up and down according to this threshold value.

\section{4 pH Sensor}

For Arduino controllers, an analog $\mathrm{pH}$ sensor is specially designed and has built-in simple, convenient and practical connection and features. It has an LED which works as the Power Indicator, a BNC connector and $\mathrm{PH} 2.0$ sensor interface. In order to use it, we simply need to connect the $\mathrm{pH}$ sensor with BNC connector, and then analog input port of Arduino controller is to plug with the $\mathrm{PH} 2.0$ interface. We will get the $\mathrm{pH}$ value easily if it is preprogrammed. It comes in compact plastic box with foams for better mobile storage. Power requirement for this sensor is $5 \mathrm{~V}$ and it is mostly used in water quality testing in agriculture and aquaculture

\subsection{ZigBee Module}

ZigBee is new wireless technology which is labeled by low rate, and low power consumption. . The zigbee module used here is zigbee S2C. The ZigBee Modules provide wireless connectivity to end-point devices in ZigBee networks. Indoor range is $30-40 \mathrm{~m}$ and outdoor range is of few hundred meters. Voltage required is $2.1 \mathrm{~V}-3.6 \mathrm{~V}$

\subsection{LabVIEW}

Laboratory Virtual Instrument Engineering Workbench (LabVIEW) is developed by National instruments. LabVIEW is a graphical user interface(GUI) software and a systemdesign platform. It is a development environment for a visual programming language . So this work which primary targets the farmers who is the layman and have scares knowledge about this computer can easily access it. Providing this comprehensive tools that you need to build any measurement or control application in dramatically less time, labVIEW is the ideal development environment for discovery, innovations and accelerated results. So, we developed a programming code in this graphical interface which could aid to farmer to interact with the system that would give the optimum utilization of the farmland. In order to develop the GUI we have use Labview as a hub which governs entire system. This work is an example of embedded system which uses software as well as hardware implementation to achieve pre specified goal.

\section{EXECUTION OF THE WORK}

The purpose system consist of four different nodes named as Node 1, Node 2, Node 3 and Receiving Node. These three nodes $1,2 \& 3$ being placed at Field station and a Receiver /coordinator Node being placed at Base station to create WSN within their range of ZigBee $\mathrm{n} / \mathrm{w}$ These Node $1,2 \& 3$ are actually responsible for acquiring real time data from the field and transmitting it and coordinator node is responsible for receiving the data. The basic building block consisting base station acting as a gateway between sensor nodes and the end user.[9 ] [10 ].

Both the Node 1 and Node 2 consists of 2 sensors each. First sensor is Temperature and Humidity Sensor and the second sensor is Soil Moisture Sensor. Node 3 consists of 3 sensors. First sensor is Temperature and Humidity Sensor, second sensor is Soil Moisture Sensor and third sensor is $\mathrm{pH}$ sensor. ALL the three nodes has Arduino uno to which sensors and ZigBee module is connected. The fourth and the final node in WSN as Coordinator/Receiver Node consists of a similar ZigBee board as in Node 1, Node 2 and Node 3 hooked up to the PC installed with labVIEW. The various steps for carrying out implementation of proposed work for creating WSN for precision farming are as follows

Step 1 Collecting real time data of temperature, humidity, soil moisture, and soil PH value in different locations in particular area of land.. Step 2 This real time data acquired is send through transmitter ZigBees placed at field station.

Step 3 This real time data is received through coordinator ZigBee placed at base station. Step 4 This real time data is graphically represented on PC using labVIEW...Step 5 Farmers can take important decisions based on real time data.

\section{RESULTS}

The results of all three transmitter nodes have been successfully obtained at coordinate node through ZigBee $\mathrm{n} / \mathrm{w}$. Here data is serially send and received by ZigBees. Serial data is obtained at coordinator node (see figure 2 ). Front Panel of LabVIEW showing graphical presentation of parameter temp, humidity and soil moisture of node 1 (see figure 3 ). Front Panel of LabVIEW showing graphical presentation of parameter temp., humidity and soil moisture and ph values of 
node 3(see figure 4) It is quite useful for taking various decisions by farmers. Here temp stands for temperature, humi stands for humidity, soil stands for soil moisture, ph stands for PH parameter, node stands for value of Node i.e 1,2 \&3.Data is extracted for each node and presented graphically. The value for soil can vary from value 1-3, value 1 mean dry soil, value 2 mean moderate soil and value 3 means wet soil. The value of ph will remain 0 for node 1 and node 2 as ph sensor is not connected to it.

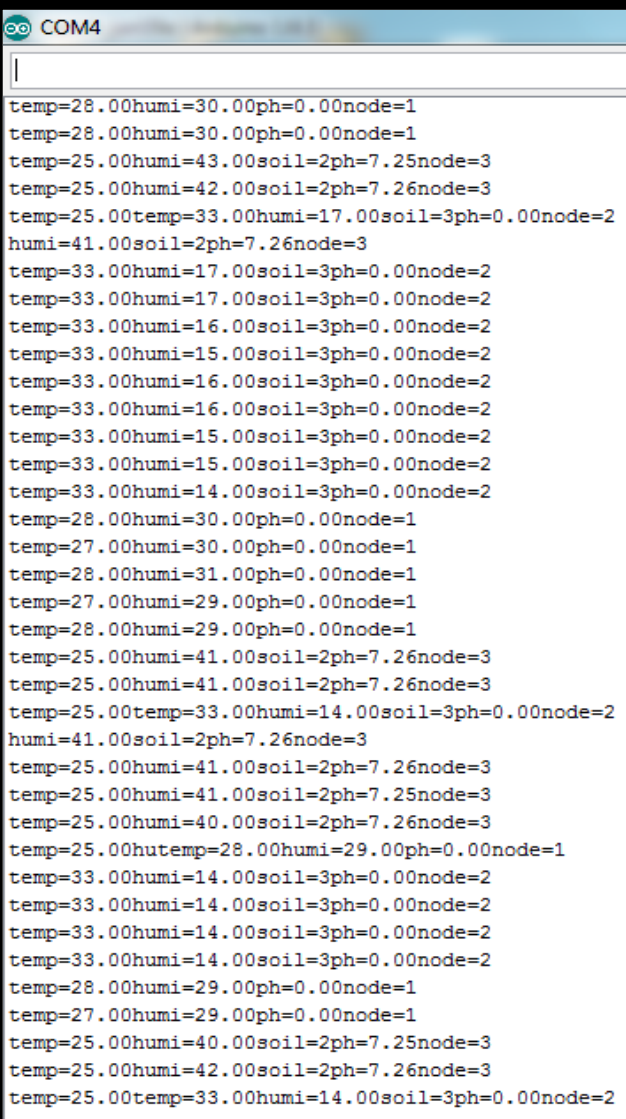

Fig 2: Reception of data at receiver node.

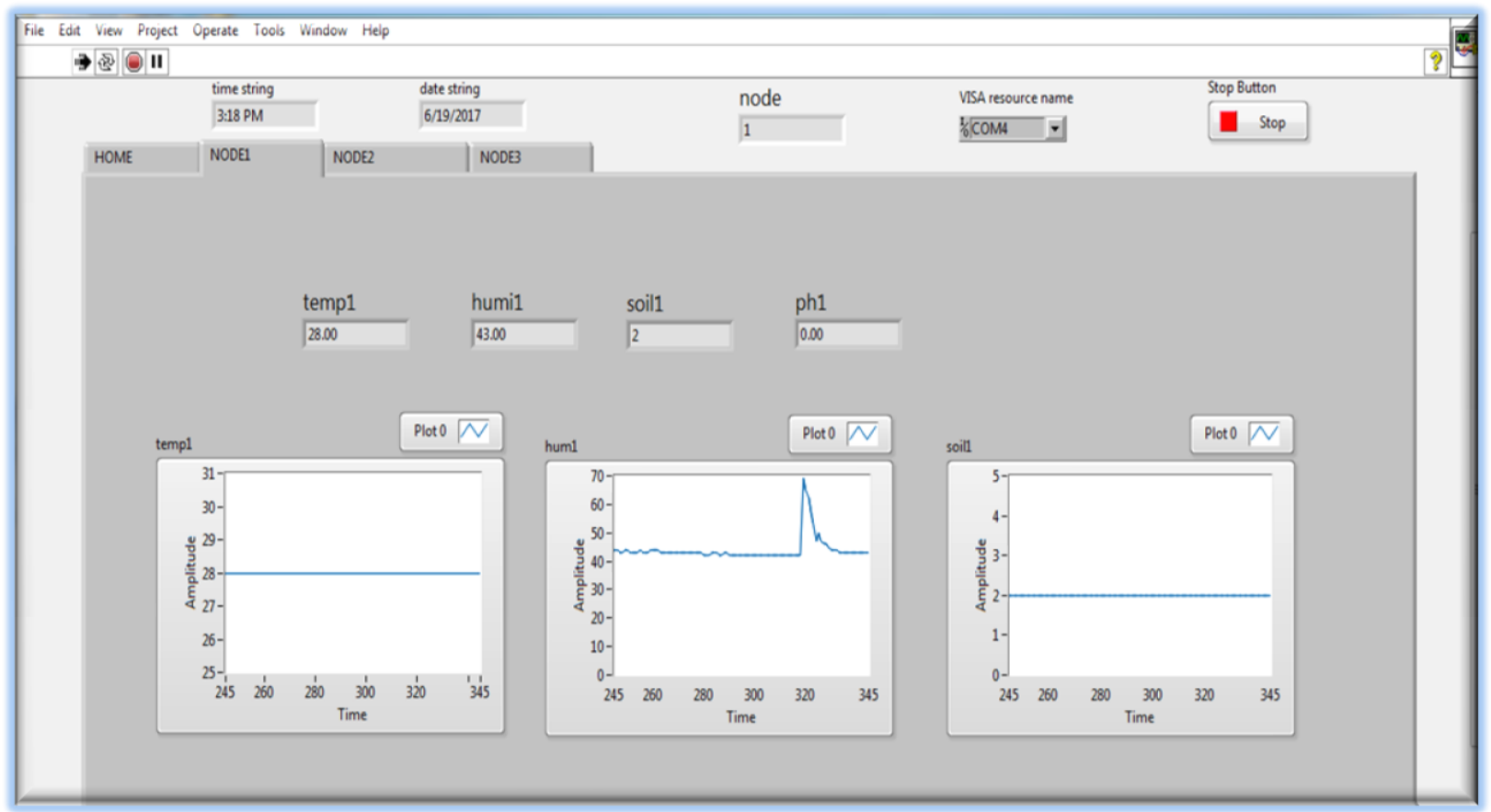

Fig 3: Front panel of LabVIEW showing results of Node1 having temperature, humidity and soil moisture values 


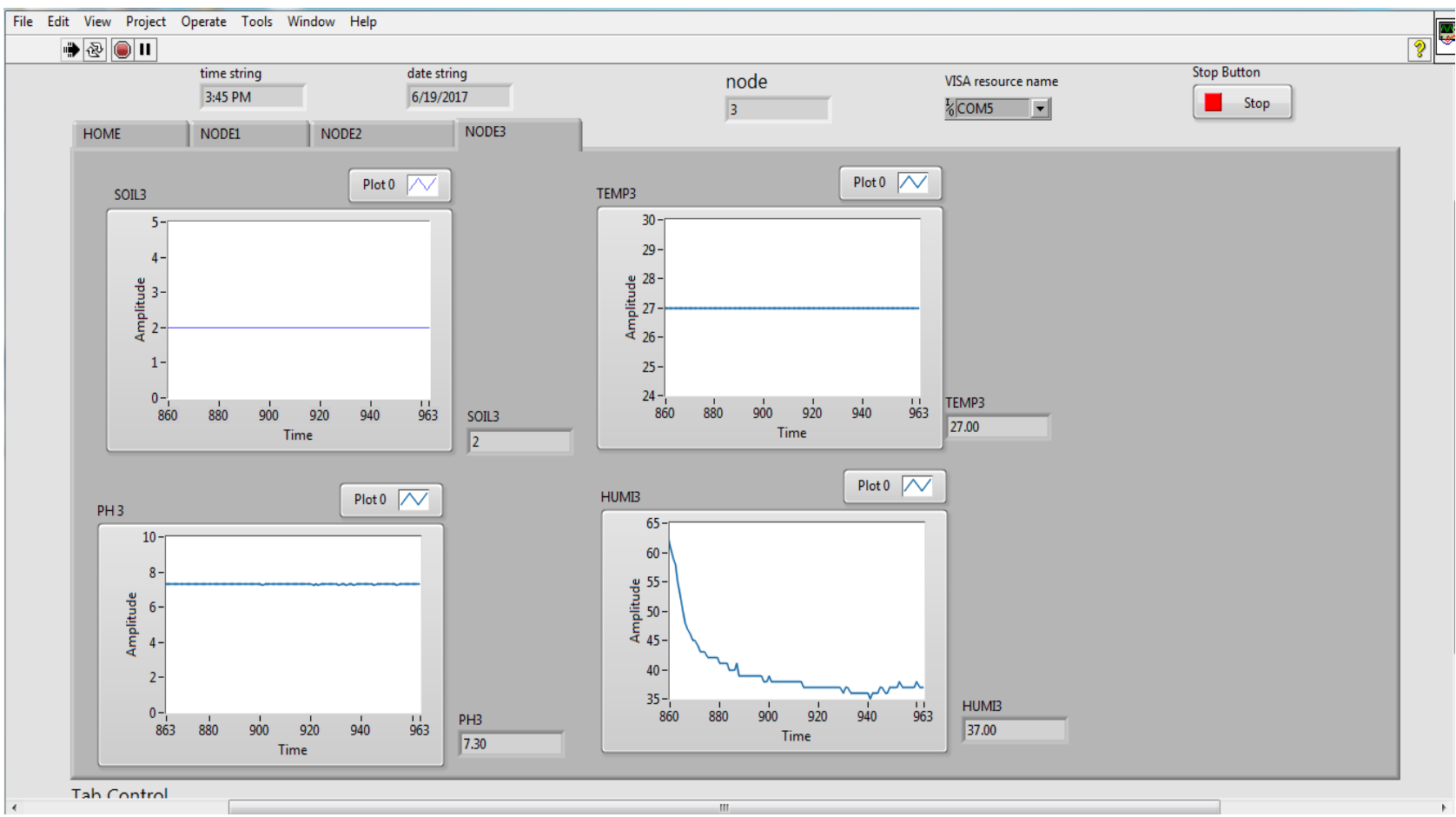

Fig 4: Front panel of LabVIEW showing results of Node 3 having temperature, humidity, soil moisture and pH sensor values

\section{CONCLUSIONS}

Precision agriculture system using LabVIEW with ZigBee based control system has been developed fruitfully. Real time monitoring system has been prove to great help to farmers for taking various type of important decisions at correct times and appropriate areas of the field. A sensing system combine with ZigBee based wireless networking has been tested to be quite effective and efficient to increase crop yield and large amount of output can be gained using limited resources and there is no wastage of resources. So, Precision irrigation system and WSN applications combines an exciting and new area of research that will greatly improve in precision farming. So, PA using LabVIEW has created scope of transforming the traditional agriculture, through the way of proper resource utilization and management, to a environmental friendly sustainable agriculture. PA not only suitable for developed countries but also for developing countries, if applied properly, and can work as a tool to destroy the distance between developed world and the rest.

\section{REFERENCES}

[1] Burrell J., Brooke T. and Beckwith R.2004. Vineyard computing: Sensor networks in agricultural production. IEEE Pervasive Computing.

[2] Zhang, W., Yong, H., Fei, L., Congcong, M. and Yuewei, C.2011 The fuzzy decision-making method of irrigation amount based on ET and soil water potential, Electronics, Communications and Control. (ICECC)

[3] Anurag D, Siuli Roy and Somprakash Bandyopadhyay.2007 Agrosense:precision agriculture using sensorbased wireless mesh networks, Indian Institute of Management Calcutta

[4] Ian, F., Akyilidiz, Weilian Su., Sankarasubramaniam, Y and Cayirci, E. 2002.Georgia Institute of technology. IEEE Communications Magazine.

[5] Mat, I., Kasim, M.R.M., and Harun, A. N. 2014. Precision irrigation performance measure using wireless sensor network. IEEE

[6] Dam, T. and Langendoen., K.2003. An Adaptive EnergyEfficient MAC Protocol for Wireless Sensor Networks. In Proceedings of SENSYS, Association for Computer Machinery.

[7] G. Lu, Krishnamachari B., and Raghavendra C. 2004. Adaptive Energy-Efficient and Low-Latency MAC for Data Gathering in Sensor Networks. In Proceedings of WMAN, Institute fur Medien Informatik.

[8] W. Ye, J. Heidemann. and D. Estrin, 2002. An EnergyEfficient MAC Protocol for Wireless Sensor Networks. In Proceedings of INFOCOM, IEEE Computer and Communications Societies.

[9] Anurag D, Siuli Roy and Somprakash Bandyopadhyay. 2008. Agro-sense: precision agriculture using sensorbased wireless mesh networks 92-61-12441-0/cfp0838e (C) itu

[10] Jzau-Sheng Lin,Chun-Zu Liu.2008. A Monitoring System Based on Wireless Sensor Network and an SoC Platform in Precision Agriculture 978-1-4244-2251$7 / 08 / \$ 25.00$ @ I E E E 